\title{
Human Interface Based on Eyelid Shape Approximation
}

\author{
Nobuaki Nakazawa, Shinnosuke Segawa, Nao Ozawa, Yuki Haruyama, Ilhwan Kim, and Yusaku Fujii
}

\begin{abstract}
This paper describes a man machine interface based on gazing input without user's body restrictions. Gazing points forward to the side directions were estimated by the relative distance of an iris and the outer corner of left and right eyes. Whether or not the user gazes the camera was recognized by the intersection of the two perpendicular bisectors derived by the position of a cornea and the eyelid edges. The suggested recognition system was applied to the operations of the page turner machine. It could judge the user's states of both reading a book and operating the machine by the special feature points on an eye.
\end{abstract}

Index Terms-Gazing, eyelid shape, bezier curve, human interface, image processing.

\section{INTRODUCTION}

In recent years, a digital book has been widely spread and it is becoming a familiar with us. While the electronic media such as a digital book are used instead of a paper book, there are also many people who like the printed books. In the welfare field, the page turner machine has been developed for enabled persons to treat the printed books such as a magazine and newspaper. A few page turner machines has already come onto the market and it is adapted to various size books and each page can be precisely turning over. The general interfaces for its operation are a contact switch or a joystick and it allows a rapid and smooth operation. However, the physical burden may give the user if these interfaces are used on the bed. The development of the interface using eyes has been done flourishingly and these apparatus attract attention as an intention transmission device for severe ALS patients now in the field of welfare. For the detection of the gaze point, the method using the EOG (Electro-oculogram) [1] and the colored contact lenses [2] are suggested. However, the EOG needs amplifier equipment and it is a lot of hassle for user to put on a contact lenses. The method by the infrared irradiation to an eyeball [3] becomes a representative, and the Purkinje image from the relative position with the pupil is used to estimate a gazing direction, but after all infrared ray irradiation equipment is necessary. On the other hand, sclera

Manuscript received July 20, 2015; revised November 12, 2015. This research was partially supported by the Ministry of Education, Science, Sports and Culture, Grant-in-Aid for Scientific Research (C), 2011-2013, No.23560282.

Nobuaki Nakazawa, Shinnosuke Segawa, Nao Ozawa, Yuki Haruyama, and Yusaku Fujii are with Division of Mechanical Science and Technology, Faculty of Science and Technology, Gunma University, Gunma, Japan (e-mail: n.nakazawa@gunma-u.ac.jp, t14802043@gunma-u.ac.jp, t15802016@gunma-u.ac.jp, t12303033@gunma-u.ac.jp, fujii@el.gunma-u.ac.jp).

Ilhwan Kim is with Department of Electrical and Electronic Engineering, Kangwon National University, Chuncheon, Korea (e-mail: ihkim@kangwon.ac.kr). reflection method using the reflectance of a sclera, an iris and the cornea is suggested under a natural light condition [4], [5]. From a viewpoint of geometric, the methods based on the oval approximation of the iris outline [6], the combination of cornea reflection image and iris outline information [7], and the cornea center of curvature position [8] have been suggested. These require the condition that an iris is taken in as an image clearly, but there are yet many problems with the aspect of the oculomotor recognition of the top and bottom direction because an iris image may hide by the movement of eyelids. This study proposes a novel method considering the eyelid shape by the Bezier curve approximation to recognize the vertical gazing directions under the natural light, without infrared rays. Gazing points forward to the side directions were derived by the relative distance of an iris and the outer corner of left and right eye. The proposed was applied to the operations of the page turner machine. It was confirmed that the developed system could successfully recognize the user's states of both reading a book and operating the machine only by gazing action.

\section{RECOGNITION OF GAZING DIRECTION}

\section{A. Measurement System}

Fig. 1 shows flow chart of the developed system. User's face image was taken to the personal computer by using USB camera(320×240pixels, RGB24, 30fps) and it was transferred to the bitmap image through the Direct Show contained in Microsoft DirectX 9c SDK. The image around the left and right eyes was picked up from the obtained overall face image by using brightness only red signal of RGB. In order to detect the special feature points, the obtained image was binarized by the following equation.

$$
g(i, j)=\left\{\begin{array}{c}
1(R[i, j]<\varepsilon) \\
0(\text { Ohterwise })
\end{array}\right.
$$

where, $R[i, j]$ shows red brightness of RGB color on the coordinate $(i, j)$ of the obtained image and $\varepsilon$ is a variable threshold for a binarization procedure. The center position of the left and right iris area, $P_{p l}\left(x_{p l}, y_{p l}\right)$ and $P_{p r}\left(x_{p r}, y_{p r}\right)$ could be expressed as follows;

$$
x_{p l / p r}=\frac{\sum_{i}\{i \cdot g[i, j]\}}{\sum_{i} \sum_{j} g[i, j]}, y_{p l / p r}=\frac{\sum_{j}\{i \cdot g[i, j]\}}{\sum_{i} \sum_{j} g[i, j]}
$$

Blink action often disturbs the recognition of special feature points. So, the blink motion was observed by the iris area calculation to eliminate error position. The position of the inner corner of the left and right eyes, $P_{h l}\left(x_{h l}, y_{h l}\right)$, 
$P_{h r}\left(x_{h r}, y_{h r}\right)$, and the position of the outer corner of the left and right eyes, $P_{c l}\left(x_{c l}, y_{c l}\right), P_{c r}\left(x_{c r}, y_{c r}\right)$ could be detected after finding the edge of the eyelid image.

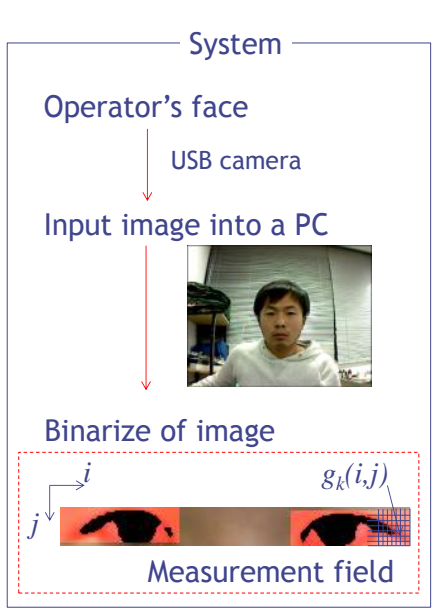

Fig. 1. Flow chart.

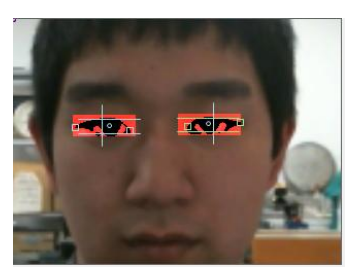

Gazing camera

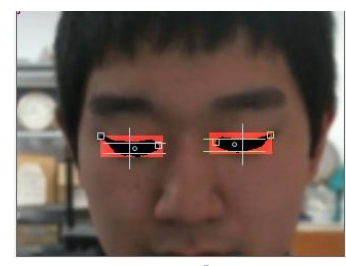

Gazing lower

Fig. 2. Eyelid shape.

\section{B. Approximation of Eyelid Shape}

As for the gazing recognition in the vertical direction, the existed methods [2]-[8] have a trouble that the eyelid seals the iris and interrupts gazing recognition. Fig. 2 shows the facial images when the human subject gazed the camera and lower position than the camera position. As shown in this figure, the iris clearly appeared in case of gazing the camera, on the other hand, the iris was hidden by the eyelid in case of lower gazing. This fact was considered as demerit for the prior method, but we focused on the shape of the eyelid. Firstly, the shape of the eyelid was approximated by using Bezier curve. The $n$ order Bezier curve was composed of $(n+1)$ control points defined as $P_{i}=\left(x_{i}, y_{i}\right)$, and the curve equation $P(u)$ can be expressed by

$$
P(u)=\sum_{i=0}^{n} B_{i}^{n}(u) P_{i}
$$

where, $B_{i}^{n}(u)$ is Bernstein polynomial given by next equation.

$$
B_{i}^{n}(u)=\frac{n}{(n-i)} u^{i}(1-u)^{u-1}
$$

where, $u$ is a parameter whose range is $[0,1]$. The Bezier curve always goes cross the edge points, starting point $P_{0}=\left(x_{0}, y_{0}\right)$ and goal point $P_{n}=\left(x_{n}, y_{n}\right)$. Here, the three order Bezier curve $(n=3)$ was applied to the eyelid shape approximation, due to the fact that it is enough to express the curve with one convex whose summit part can be located at everywhere, while keeping both edge points, as shown in Fig. 3. The three order Bezier curve can be given by

$$
\begin{aligned}
B_{x}(u)= & \left(x_{0}-3 x_{1}+3 x_{2}-x_{3}\right) u^{3} \\
& +\left(3 x_{1}-6 x_{2}+3 x_{3}\right) u^{2}+\left(3 x_{2}-3 x_{3}\right) u+1 \\
B_{y}(u)= & \left(y_{0}-3 y_{1}+3 y_{2}-y_{3}\right) u^{3} \\
& +\left(3 y_{1}-6 y_{2}+3 y_{3}\right) u^{2}+\left(3 y_{2}-3 y_{3}\right) u+1
\end{aligned}
$$

As shown in Fig. 4(a), the inner and outer corner of the eye were assigned to the edge points, $P_{0}=\left(x_{0}, y_{0}\right)$ and $P_{3}=\left(x_{3}, y_{3}\right)$. Other two control points were put on the extension of a $y$ directional straight passing through the edge point of the iris in the $x$ axis. These two control points were determined by decreasing the error between the eyelid curve and the Bezier curve. The curvature of approximated Bezier curve can be given by

$$
K=\frac{B_{x}^{\prime}(u) B_{y}^{\prime \prime}(u)-B_{\mathrm{x}}^{\prime \prime}(u) B_{y}^{\prime}(u)}{\left[\left\{B_{x}^{\prime}(u)\right\}^{2}+\left\{B_{y}^{\prime}(u)\right\}^{2}\right]^{3 / 2}}
$$

Here, the curvature on the middle point $(u=0.5)$ from starting point to goal point was utilized for gazing recognition of vertical direction.
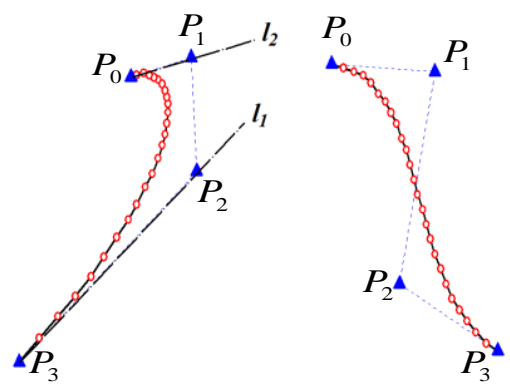

Fig. 3. Bezier curves.

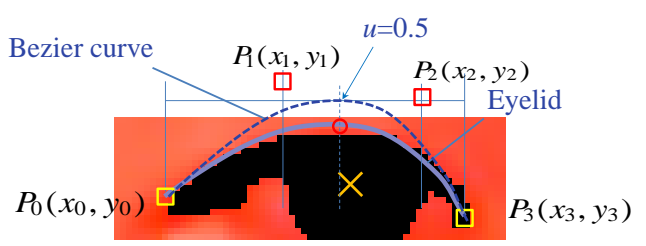

(a) Approximation by Bezier curve

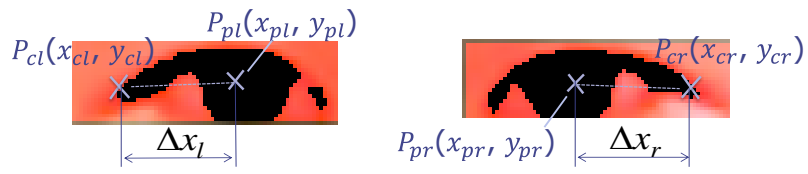

(b)Relative position between both eyes

Fig. 4. The special feature points.

\section{A. Relative Position between Both Eyes}

With reference to the detection of gazing in the side direction, the positions of both iris and the outer of the eye were used. As shown in Fig. 4(b), the gazing in the side direction was detected by

$$
R=\frac{\left|x_{p r}-x_{c r}\right|}{\left|x_{p l}-x_{c l}\right|}=\frac{\Delta x_{r}}{\Delta x_{l}}
$$

where, $x_{c l}$ and $x_{c r}$ are left and right corner position of the eye, $x_{p l}$ and $x_{p r}$ are left and right iris in the $x$ direction, as shown in this Figure. The length of $\Delta x_{l}$ and $\Delta x_{r}$ are different according to each person, therefore, the calibration process is needed for eyes recognition. We could eliminate the calibration process due to the fact that the parameter $R$ was normalized by using both left and right iris positions.

\section{B. Experiment}

In order to investigate the characteristics of the ratio $R$ and the curvature $K$, the gazing experiment was carried out. As shown in Fig. 5, subject was lying on the back to gaze the board on which sixteen images $(4 \times 4)$ were put. The subject 
was directed to gaze each picture from upper left to lower right in order for ten seconds per one image. The USB camera was put on the upper position of the board to observe the subject's eyes. The time trajectories of (7) and (8) are shown in Fig. 5. The numbers of this figure are corresponding to the image numbers shown in Fig. 6. With reference to the ratio $R$, it was increasing with step as the subject's gazing point was moved to the right direction. As for the curvature $K$, its value had four steps and it was found that the curvature of eyelid included the gazing point information with respect to the vertical directions. The developed system was utilized as a human interface for page turner machine.

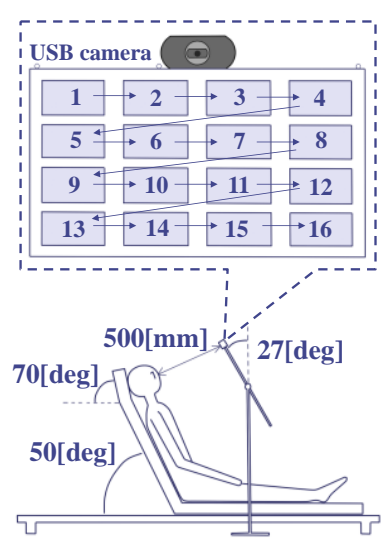

Fig. 5. Experiment.

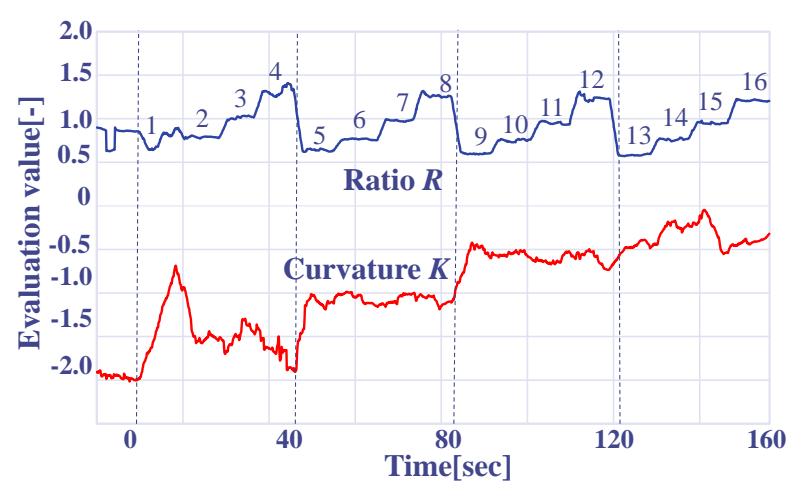

Fig. 6. Time trajectories of (7) and (8).

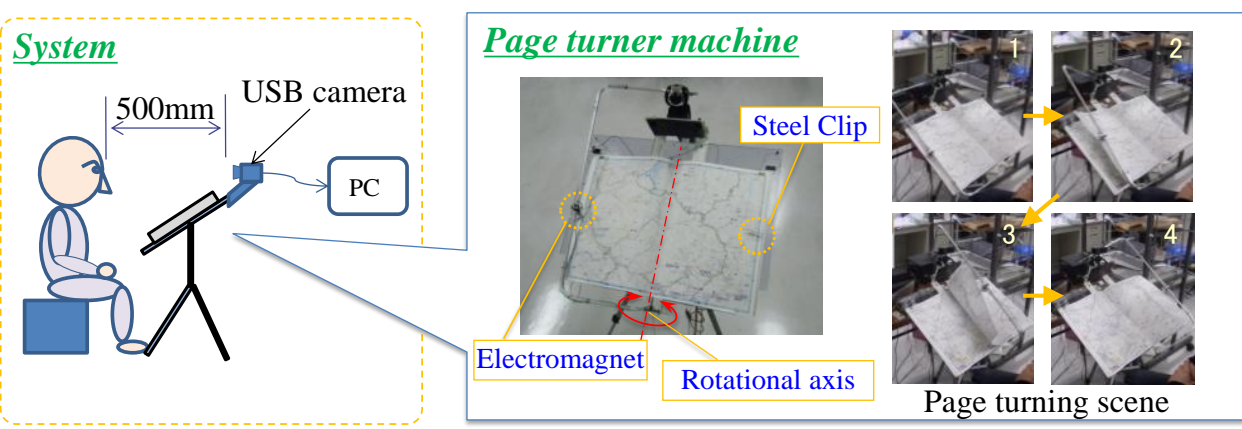

Fig. 7. Page turner machine.

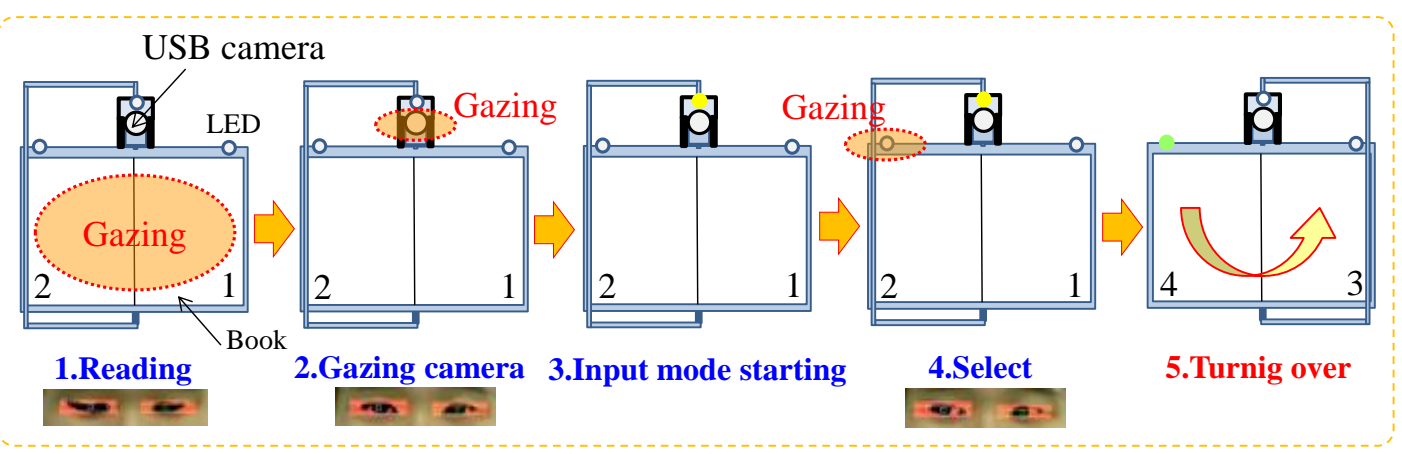

Fig. 8. Operation method.

\section{APPLICATION}

\section{A. Page Turner Machine}

Page turner machine has been used for enabled persons who cannot use their hands as welfare support equipment, so as to turn over a page of book automatically by something input. Fig. 7 shows the page turner system. The equipment was composed of flat sloped board to put a book, USB camera, page turner arm, and it was supported by a tripod. The arm was connected to the DC servo which had a reduction gear (Reduction gear ratio is 1/230), and it could rotate around the axis. The clip was put on each page of the book and the electromagnet mounted on the rotation arm picked it up to turn over each page. The USB camera in front of user observed the gazing actions so as to reflect the user's intention on the operation of the page turner machine. The DC motor and the electro magnet were controlled by the personal computer via the microcomputer and the motor driver. In addition, Three LED put on the upper part of the sloped board could inform the control states to the user.

\section{B. Operation Method}

Based on the obtained results, the gazing actions were assigned to the operation of the page turner machine. Fig. 8 shows the operation method. We used four states, "gazing camera", "Left LED gazing", "Right LED gazing", and “ reading a book". In cases where user was reading a book (State 1), it is corresponding to gazing lower than the USB camera and the system was static situation. When the user 
gazed the USB camera for more than two seconds (State 2), the system recognized the state of "gazing camera" and the center LED near the USB camera was turned on (State 3). In this state, the system was waiting for the user's something input. If the user gazed the left side or the right side LEDs for more than $2 \mathrm{sec}$, they illuminated to inform an input sign to the user (State 4) and the machine consequently started to turn over a page of the book (State 5).

\section{Test Trial}

Test operation was carried out to verify the proposed method. In the verification experiment, "gazing camera" was recognized in the case of $K>1.5$, and in other cases "reading a book" was recognized. When the circumstance was satisfied with $R<0.75$ and $R>1.25$, the system recognized the left and right LED, respectively. Subject sat on the chair apart from $500[\mathrm{~mm}]$ shown in Fig. 7, and operated the page turner machine by gazing actions. The subject was firstly reading a book, and after the experimenter's sign, the operation was started by gazing the USB camera mounted on the page turner machine. In the experiment, left and right page turnings were carried out for ten times. Five subjects participated in the experiment. Table I shows the experimental results of operating the page turner machine by gazing actions. The values in this table are recognition times of ten trials about "reading a book", "gazing camera", "Turning left page", and "Turning right page". With reference to "reading a book" and "gazing camera", all trials were recognized and all five subjects could consequently move reading state into operating state. As for the operation, most trials of turning over a page were successfully carried out. However, a few misrecognitions could be seen in the trial of subject C and D. This is because the correct positions of the outer corner of the eye could not be observed due to the fact that the procedure range often included the subject's hair, and this problem is considered as a future works to modify the developed system.

TABLE I: SUCCESSFUL TIMES OF 10 TRIALS

\begin{tabular}{|c||c|c|c|c|}
\hline & Reading & Gazing camera & Left turning & Right turning \\
\hline \hline A & $\mathbf{1 0}$ & $\mathbf{1 0}$ & $\mathbf{1 0}$ & $\mathbf{1 0}$ \\
\hline B & $\mathbf{1 0}$ & $\mathbf{1 0}$ & $\mathbf{1 0}$ & $\mathbf{1 0}$ \\
\hline C & $\mathbf{1 0}$ & $\mathbf{1 0}$ & 9 & $\mathbf{1 0}$ \\
\hline D & $\mathbf{1 0}$ & $\mathbf{1 0}$ & 9 & 8 \\
\hline E & $\mathbf{1 0}$ & $\mathbf{1 0}$ & $\mathbf{1 0}$ & $\mathbf{1 0}$ \\
\hline
\end{tabular}

\section{CONCLUSION}

This paper proposed a gazing interface based on the special feature points of an eye. The existed methods have a problem that the eyelid seals the iris in the lower directional gazing with respect to the camera and interrupts gazing recognition. Here, we paid attention to the eyelid shape to detect gazing actions in the vertical direction and applied the Bezier curve to shape approximation so as to derive the eyelid curvature. Gazing points forward to the side directions were derived by the relative distance of an iris and the outer corner of left and right eye. The proposed recognition system was applied to the operations of the page turner machine. It was confirmed that the developed system could successfully recognize the user's states of both reading a book and operating the machine only by gazing action. As a future work, we would like to enhance the position recognition of the special feature point, such as the outer corner of the eye by eliminating the influence of the user's hair.

\section{REFERENCES}

[1] N. Itakura, T. Oota, and K. Sakamoto, "Investigation for calculation method of eye-gaze shift from electro-oculograph amplified by AC coupling with using eye-gaze input interface," Transactions of the Institute of Electronics, Information and Communication Engineers D, vol. J90-D, no. 10, pp. 2903-2913, 2007.

[2] N. Nishiuchi, H. Takada, and K. Kurihara, "A study on non-contact line-of-sight detection using color contact lens," Transactions of the Japan Society of Mechanical Engineers, Series(C), vol. 65, no. 636, pp. 3314-3320, 1999.

[3] Y. Ebisawa and A. Nakashima, "Increasing precision of pupil position detection using the corneal reflection," Journal of the Institute of Image Information and Television Engineers, vol. 62, no. 7, pp. 1122-1126, 2008.

[4] K. Abe, M. Oouchi, S. Ooi, and M. Ooyama, "Eye-gaze input system based on the limbus tracking method using image analysis," Journal of the Institute of Image Information and Television Engineers, vol. 57, no. 10 , pp. $1354-1360,2003$

[5] T. Takegami, T. Gotoh, and G. Ooyama, "An algorithm for model-based stable pupil detection for eye tracking system," Transactions of the Institute of Electronics, Information and Communication Engineers D-II, vol. J86-D-II, no. 2, pp. 252-261, 2003.

[6] N. Nishiuchi, H. Takada, and K. Kurihara, "A study of non-contact line-of-sight detection using ellipse fitted to Iris edge," Transactions of the Japan Society of Mechanical Engineers, Series (C), vol. 69, no. 682, pp. 1611-1617, 2003.

[7] T. Takegami and T. Gotoh, "Vision-based algorithm for eye movement detection using corneal reflection and Iris contour," Transactions of the Institute of Electronics, Information and Communication Engineers D-I, vol. J82-D-I, no. 10, pp. 1295-1303, 1999.

[8] H. Tanaka, S. Hikita, T. Kasai, and T. Takeda, "Eye-gaze detection based on the position of the cornea curvature center using two near-infrared light sources," Technical Report of the Institute of Electronics, Information and Communication Engineers, vol. 108, no. 479, pp. 177-180, 2009.

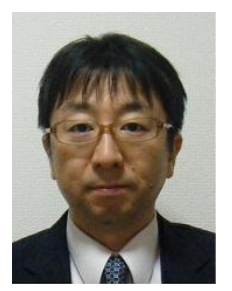

Nobuaki Nakazawa was born in 1969, Japan. He received the B.S. degree from Toyama University in 1993, and M.S and Dr. Eng. degrees from Tohoku University, Japan in 1995 and 1998, respectively. From 1998 to 1999, he was a Research Fellow of Japan Society for the Promotion Science (JSPS) at Tohoku University. From 1999 to 2006, he was a Research Associate of Graduate School of Engineering, Gunma University, Japan. Since 2007, he has been an Associate Professor of the Department of Production Science and Technology, Gunma University, Japan. His research interests include ergonomics, human interface, and welfare support-equipment.

Shinnosuke Segawa was born in 1991, Japan. He received the B.S. degree

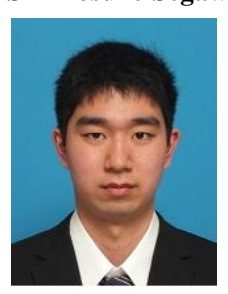
from Gunma University, Japan in 2013. He is now a Master Course Student of Gunma University in Japan. His research interests include ergonomics and human interface.

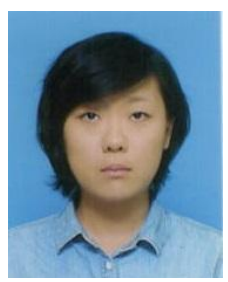

Nao Ozawa was born in 1992, Japan. She received the B.S. degree from Gunma University, Japan in 2014. She is now a Master Course Student of Gunma University in Japan. Her research interests include mechatronics and human interface. 


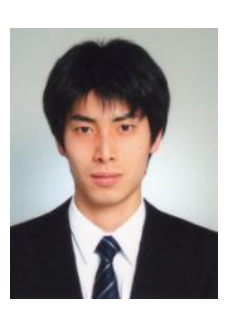

Yuki Haruyama was born in 1993, Japan. From 2012, he is an under graduate course student of the Department of Production Science and Technology, Gunma University, Japan. His research interests include software and image processing.

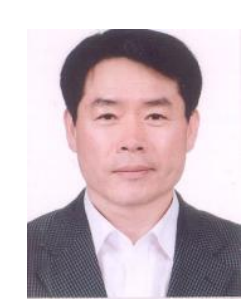

Il-Hwan Kim was born in 1959, Korea. He received the B.S. and M.S. degrees in control and instrument engineering from Seoul National University in 1982 and 1985 respectively, and the Ph.D. at the Tohoku University, Japan in 1993. In 1995, he joined the Department of Electrical and Computer Engineering at Kangwon National University and is currently a Professor. His research interests include control, mechatronics, and human interfaces.

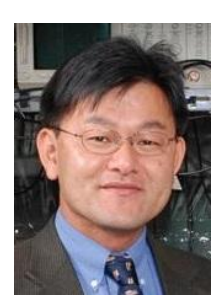

Yusaku Fujii was born in Tokyo, Japan, in 1965. He received the B.E., M.E. and Ph.D degrees from Toyko University, Tokyo, Japan, in 1989, 1991 and 2001, respectively. In 1991, he joined the Kawasaki Steel Corp. In 1995, he moved to the National Research Laboratory of Metrology (NRLM), Tsukuba, Japan, where he had studied for the replacement of the kilogram using the Superconducting Magnetic Levitation. In 2002, he moved to Gunma University, Kiryu, Japan, where has invented and studied for the Levitation Mass Method (LMM) as a precision force measurement method. He has also invented and studied for the e-JIKEI Network as a security camera system with privacy protection (www.e-jikei.org). 